\title{
Malignant Glomus Tumor of the Peritoneum: Case Report
}

\author{
Sandra Baleato-González, MD, PhD ${ }^{1}$, Roberto García-Figueiras, $M D^{1}$, Maria Virginia Trujillo-Ariza, $M D^{1}$, \\ Juan Jose Carrera-Álvarez, MD² \\ Departments of ${ }^{1}$ Radiology and ${ }^{2}$ Pathology, Complexo Hospitalario Universitario de Santiago de Compostela, Choupana s/n, 15701 Santiago de \\ Compostela (A Coruña), Spain
}

Glomus tumors are usually benign tumors that occur in the skin and soft tissues of the extremities. Visceral locations, such as stomach, intestines or lung, are extremely rare because glomus bodies are rare or absent in these organs. This report describes our experience in a 47-year-old woman diagnosed with a peritoneal malignant glomus tumor. This finding has not been previously reported.

Index terms: Peritoneal tumors; Malignant glomus tumor; Glomangiosarcoma

\section{INTRODUCTION}

Glomangiosarcoma or malignant glomus tumor is a very rare neoplasm that usually arises from the glomus body. The glomus body is a form of arteriovenous anastomosis surrounded by glomus cells (modified smooth muscle cells), which plays an important role in thermoregulation. Neoplasms of the glomus organ are most commonly encountered in the skin and soft tissues of the extremities, particularly the subungual region. However, diverse unusual locations have been reported that include the chest, abdominal wall, bone, stomach, eyelid, nose and mediastinum. This article describes a case of peritoneal

Received May 22, 2013; accepted after revision October 10, 2013. Corresponding author: Sandra Baleato-González, MD, PhD, Department of Radiology, Complexo Hospitalario Universitario de Santiago de Compostela, Choupana s/n, 15701 Santiago de Compostela (A Coruña), Spain.

- Tel: (34) 981950843 • Fax: (34) 981706198

- E-mail: baleatorum@hotmail.com

This is an Open Access article distributed under the terms of the Creative Commons Attribution Non-Commercial License (http://creativecommons.org/licenses/by-nc/3.0) which permits unrestricted non-commercial use, distribution, and reproduction in any medium, provided the original work is properly cited. glomangiosarcoma. To our knowledge, has not been reported in the radiological literature.

\section{CASE REPORT}

A screening gynecological exam was performed in a 47-year-old woman. The bimanual pelvic exam revealed the presence of bilateral parauterine masses. These masses were partially mobile and poorly demarcated. Transvaginal ultrasound showed a right parauterine mass of $11.9 \mathrm{x}$ $4.7 \mathrm{~cm}$ and a left one of $13.2 \times 7.9 \mathrm{~cm}$. The masses were heterogeneous with irregular margins and were barely vascularized, which encompassed the left ovary and fallopian tubes. No free liquid was observed. A double phase contrast-enhanced multidetector computed tomography (CT) scan was performed with the intravenous injection of 120 $\mathrm{mL}$ non-ionic contrast medium at flow rate of $4 \mathrm{~mL} / \mathrm{sec}$. The CT exam, which included a late arterial phase of the thorax and abdomen followed by a portal phase of the abdomen and pelvis, revealed a poorly defined mass occupying the inframesocolic peritoneal space and both paracolic gutters. This mass showed extensive low-density areas (with CT density values around 25 Hounsfield units [HU]) with interspersed foci of higher attenuation (approximately 


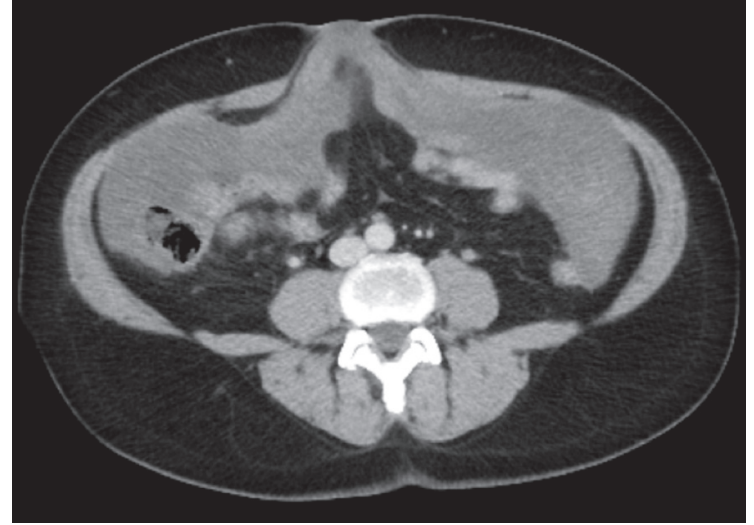

A

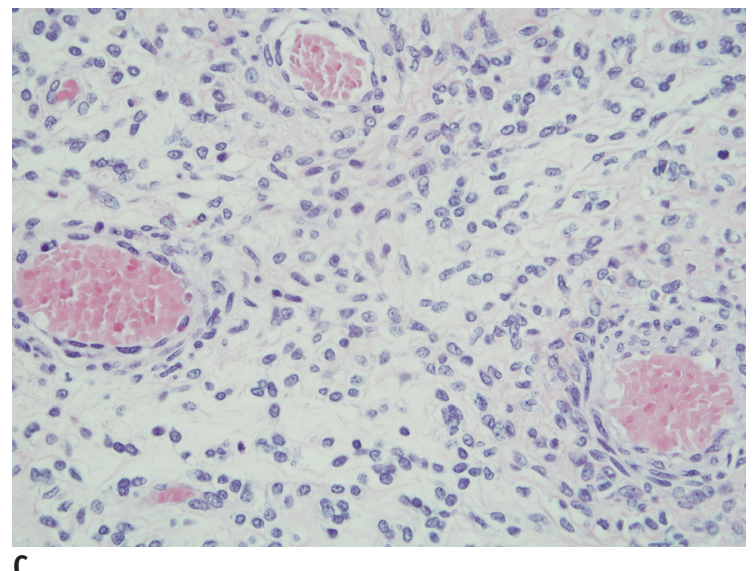

C

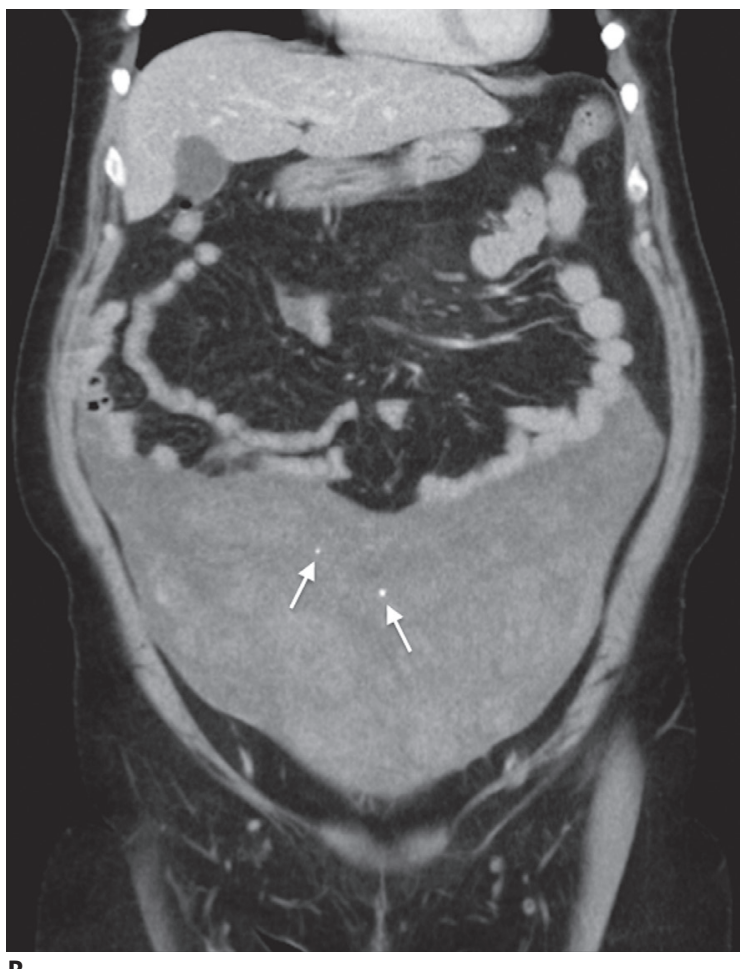

B

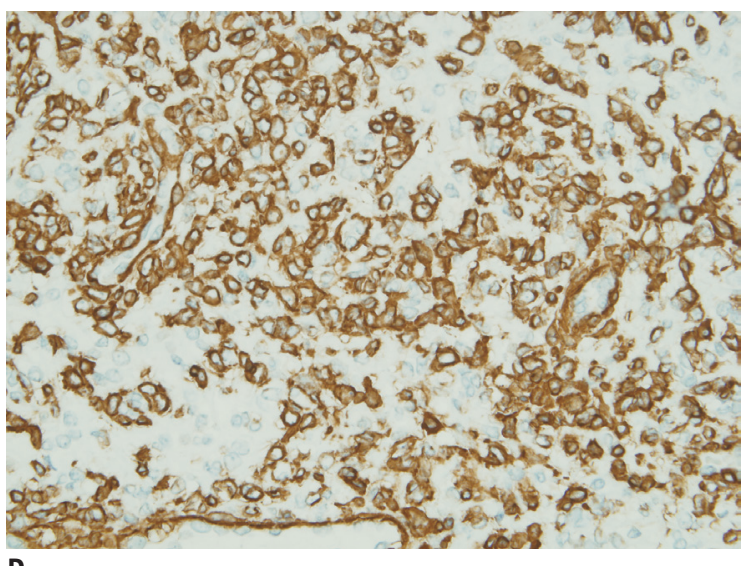

D

Fig. 1. CT and staining observations.

A. Axial CT after contrast administration in portal phase shows large mass that involved peritoneal surface without visceral organ affected. B. Coronal reformatted CT after contrast administration in portal phase demonstrates inframesocolic and paracolic gutters mass with heterogeneous enhancement and few small foci inside (arrows). C. Hematoxylin and eosin stain (20 x) shows glomus body consisting of uniform small, rounded cells with centrally placed round and pleomorphic nuclei. These cells are located around vessels. D. Smooth muscle actin immunostain demonstrating strong cytoplasmic positivity.

$50 \mathrm{HU}$ ) and some small calcifications (Fig. 1A, B). The CT examination did not reveal any significant changes in tumor density between both phases of the acquisition. Laboratory tests evidenced high levels of CA125 antigen. An exploratory laparotomy was performed. Intraoperative findings demonstrated a poor-defined abdominopelvic soft tissue mass with extensive areas of necrosis attached to the parietal peritoneum, the visceral peritoneum of the uterine fundus, terminal ileum loops and umbilicus. The remainder of the abdominal cavity including the gastrointestinal tract and ovaries was normal. Macroscopically, the resected specimen was a large peritoneal tumor measuring $45 \mathrm{x}$ $30 \mathrm{~cm}$ in diameter, with a smooth surface, shiny, very congestive and hemorrhagic. After cutting, heterogeneous brittle and frayed hemorrhagic areas were identified without a definite pattern. Microscopically, the mass had abundant capillary-sized vessels, which had small round to dilated endothelium-line lumina surrounded by uniform sheet-like 
epitheloid tumor cells. Immunohistochemically, vimentin, (alpha)-smooth muscle actin, HHF-35 actin and collagen IV were stained intensely (Fig. 1C, D). Tumor cells were negative for desmin, CD31, CD34, cytokeratin AE1/AE3, melan-A and HMB 45. Finally, the pathologic diagnosis was peritoneal GMS. One year later, the patient remains asymptomatic without radiologic evidence of tumoral recurrence.

\section{DISCUSSION}

Glomus tumors are relatively uncommon, constituting < $2 \%$ of soft-tissue tumors. They can present as solitary or multicentric lesions (1). Histologically they resemble or are derived from cells of the neuromyoarterial glomus or glomus body, an anatomic structure thought to be involved with temperature regulation. Ultrastructurally, glomocytes have features of smooth muscle and may derive from the epithelioid neuromuscular cells associated with the arteriovenous anastomoses of the normal glomus body (2). Usually, they are located in the deep dermis or subcutis of an upper or lower extremity, but the tumor may also arise in sites where the normal glomus body may be sparse or even absent. Although the most common site is the subungual area of the finger, infrequently they involve unusual sites including the patella, the mediastinum, respiratory and gastrointestinal tract, and mesentery (3-5). Intraabdominal locations are relatively rare and peritoneal glomus tumors have seldom been reported $(6,7)$. To our knowledge, this is the first description of a malignant glomus tumor in this location.

From a clinico-pathological point of view, glomus tumors have been subclassified into benign solitary, multiple familial and malignant glomus tumors, which were subdivided into three categories: locally infiltrative glomus tumor (benign tumor but with infiltrative borders), glomangiosarcoma arising from a benign glomus tumor (sarcomatous area within a benign glomus tumor) and glomangiosarcoma de novo (undifferentiated malignant small round cell neoplasm, whose architecture and cell population resemble those of a benign glomus tumor) (4, 8). Glomangiosarcoma de novo is a difficult diagnosis and requires the recognition of histologic or ultrastructural features reminiscent of glomus tumors. Additionally, it demonstrates malignant histologic features including cytologic atypia, necrosis, elevated mitotic rate and characteristically cystic degeneration (9). Folpe et al. (10) proposed the following criteria for malignant glomangiosarcoma: tumors with a deep location and a size of more than $2 \mathrm{~cm}$, or atypical mitotic figures or marked atypia with mitotic activity. These characteristics were notably related with the risk of metastases. To the contrary, a recent article opined that these criteria can not be applied in abdominal glomus tumors and, as evidence, presented a case report of a large mesentery glomangioma without malignant features (5). Glomangiosarcoma is usually an indolent tumor but with an increased risk of local recurrence. However, metastatic dissemination has been reported $(10,11)$. Resection is the treatment of choice and, even with described metastases, patients have an excellent prognosis. Nevertheless, intrabdominal tumors are bizarre and the malignant potential of these tumors remains unclear.

Glomus tumors usually enhance strongly on early-phase contrast material-enhanced images, which reflects their hypervascular nature (12). However, hypervascularized malignant glomus tumors of the abdomen have been rarely reported (4). In our case, radiologic findings were unspecific, demonstrating a poor-enhanced low-density peritoneal tumor. Differential diagnosis should consider several primary and secondary peritoneal pathologic entities $(13,14)$, with pseudomyxoma peritonei being the first consideration. Pseudomyxoma refers to the intraperitoneal accumulation of a gelatinous ascites secondary to rupture of a mucinous tumor. This entity may produce similar imaging findings to our case. Mucinous material covers the peritoneal surface and produces scalloping of the serosal marging of the liver or spleen. Peritoneal mucinous content usually presents with low attenuation on $\mathrm{CT}$ images with areas of high attenuation due to the presence of solid elements in the mucinous material, fibrosis and compressed mesentery $(14,15)$. Tumor calcification is also a usual finding. Many authors suggested that pseudomyxoma should be divided into two categories: disseminated peritoneal adenomucinosis and peritoneal mucinous carcinomatosis (16). The first category contains benign or borderline-appearing epithelial cells or cells from welldifferentiated mucinous carcinomas. The majority of cases of classic pseudomyxoma peritonei from this first category arise in the appendix (17). Mucus-forming appendiceal lesions range from relatively benign mucosal hyperplasia to mucinous cystadenocarcinomas. Tumor invasion or appendiceal wall breakage results in intraperitoneal spread of mucin-producing tumor cells (18). On the other hand, 
peritoneal mucinous carcinomatosis is an invasive form of dissemination of a mucinous carcinoma, which originates from mucinous tumors in the ovary, gastrointestinal tract, gallbladder, pancreas or urachus. Imaging differentiation of the classic pseudomyxoma peritonei from mucinous carcinomatosis is difficult. Mucinous carcinomatosis tends to invade into parenchymal organs, more frequently involves the chest and omentum and may also be accompanied by metastatic lymphadenopathy (19).

Mesothelial tumors (peritoneal malignant mesotheliomas and well-differentiated papillary mesothelioma) may show some similar radiological manifestations to this case of glomangiosarcoma $(13,14,20,21)$. Malignant mesotheliomas may also cause a diffuse involvement of the peritoneal cavity with scalloping or direct invasion of adjacent abdominal organs. Moreover, the sarcomatous subtype of malignant primary mesothelioma may have detectable calcifications. However, imaging findings are usually quite different to our case, with two main patterns of malignant mesothelioma reported (20). The dry type is the most common, consisting of peritoneal enhancing solid masses with no signs of ascites. On the contrary, the wet type is associated with ascites and tiny peritoneal nodules and plaques. Calcification within peritoneal malignant mesothelioma is an unusual finding and these tumors occur predominantly in older men with a history of asbestos exposure (13), which was not germane in the present case. Well-differentiated papillary mesothelioma is a rare type of mesothelioma that occurs most commonly in young women without a history of asbestos exposure $(20,21)$. Peritoneal calcifications secondary to the abundant psammoma bodies within the tumor plaques have been reported in this entity. However, previously reported imaging findings include ascites, thickening and nodularity of the peritoneum and mesentery and peritoneal solid masses $(20,21)$, which are not concordant with those in our case. Finally, imaging characteristics of this case were quite different from the typical findings of several usual entities that may involve the peritoneum, such as peritoneal carcinomatosis or primary peritoneal serous carcinomas, which typically present with massive ascites without sizable mass $(13,14)$. Besides, a primary tumor is usually identified on imaging in patients with carcinomatosis (14). Other causes of peritoneal involvement, such as mesenteric lymphomatosis or tuberculosis, could be considered. However, the lack of their characteristic imaging findings (such as associated enlarged lymph nodes in lymphomatous involvement or smooth peritoneal thickening, mesenteric lymphadenopathy with central necrosis, or ascites with high attenuation in the case of peritoneal tuberculosis) can be useful for the differential diagnosis (14).

In conclusion, multiple entities can affect the peritoneum with a broad differential diagnosis. Glomangiosarcoma of the peritoneum has never been described before. Imaging findings of this tumor in this location are unspecific and pathologic confirmation is required for the final diagnosis.

\section{REFERENCES}

1. Gaertner EM, Steinberg DM, Huber M, Hayashi T, Tsuda N, Askin FB, et al. Pulmonary and mediastinal glomus tumors-report of five cases including a pulmonary glomangiosarcoma: a clinicopathologic study with literature review. Am J Surg Pathol 2000;24:1105-1114

2. Kayal JD, Hampton RW, Sheehan DJ, Washington CV. Malignant glomus tumor: a case report and review of the literature. Dermatol Surg 2001;27:837-840

3. Choi YJ, Yang KH, Gang SJ, Kim BK, Kim SM. Malignant glomus tumor originating in the superior mediastinum: an immunohistochemical and ultrastructural study. J Korean Med Sci 1991;6:157-163

4. Rodríguez-Justo M, Aramburu-González JA, Santonja C. Glomangiosarcoma of abdominal wall. Virchows Arch 2001;438:418-420

5. de Bruin AF, Verhoef C, den Bakker MA, van Geel A. Glomus tumor of the mesentery with atypical features: a case report. Int J Surg Pathol 2008;16:440-442

6. Harper L, Lavrand F, Le Bail B, Brun M, Ferron S, Oses $P$, et al. Glomus tumor of the mesocolon. J Pediatr Surg 2005;40:e37-e38

7. Alam K, Maheshwari V, Sabir F, Haq ME, Siddiqui FA, Mefuzuddin S. Glomus tumor of lesser omentum--a case report. Indian J Pathol Microbiol 2007;50:543-544

8. Hegyi L, Cormack GC, Grant JW. Histochemical investigation into the molecular mechanisms of malignant transformation in a benign glomus tumour. J Clin Pathol 1998;51:872-874

9. Hiruta N, Kameda N, Tokudome T, Tsuchiya K, Nonaka H, Hatori T, et al. Malignant glomus tumor: a case report and review of the literature. Am J Surg Pathol 1997;21:1096-1103

10. Folpe AL, Fanburg-Smith JC, Miettinen M, Weiss SW. Atypical and malignant glomus tumors: analysis of 52 cases, with a proposal for the reclassification of glomus tumors. Am J Surg Pathol 2001;25:1-12

11. De Chiara A, Apice G, Mori S, Silvestro G, Losito SN, Botti G, et al. Malignant glomus tumour: a case report and review of the literature. Sarcoma 2003;7:87-91

12. Baek HJ, Lee SJ, Cho KH, Choo HJ, Lee SM, Lee YH, et al. Subungual tumors: clinicopathologic correlation with US and MR imaging findings. Radiographics 2010;30:1621-1636

13. Levy AD, Arnáiz J, Shaw JC, Sobin LH. From the archives of 
the AFIP: primary peritoneal tumors: imaging features with pathologic correlation. Radiographics 2008;28:583-607; quiz 621-622

14. Levy AD, Shaw JC, Sobin LH. Secondary tumors and tumorlike lesions of the peritoneal cavity: imaging features with pathologic correlation. Radiographics 2009;29:347-373

15. Sulkin TV, O'Neill H, Amin AI, Moran B. CT in pseudomyxoma peritonei: a review of 17 cases. Clin Radiol 2002;57:608-613

16. Ronnett BM, Zahn CM, Kurman RJ, Kass ME, Sugarbaker PH, Shmookler BM. Disseminated peritoneal adenomucinosis and peritoneal mucinous carcinomatosis. A clinicopathologic analysis of 109 cases with emphasis on distinguishing pathologic features, site of origin, prognosis, and relationship to "pseudomyxoma peritonei". J Surg Pathol 1995;19:13901408
17. Pai RK, Longacre TA. Pseudomyxoma peritonei syndrome: classification of appendiceal mucinous tumours. In: Ceelen WP, ed. Peritoneal Carcinomatosis: A Multidisciplinary Approach. New York: Springer, 2007:71-107

18. Smeenk RM, Bruin SC, van Velthuysen ML, Verwaal VJ. Pseudomyxoma peritonei. Curr Probl Surg 2008;45:527-575

19. Bechtold RE, Chen MY, Loggie BW, Jackson SL, Geisinger K. CT appearance of disseminated peritoneal adenomucinosis. Abdom Imaging 2001;26:406-410

20. Park JY, Kim KW, Kwon HJ, Park MS, Kwon GY, Jun SY, et al. Peritoneal mesotheliomas: clinicopathologic features, CT findings, and differential diagnosis. AJR Am J Roentgenol 2008;191:814-825

21. Herregods N, Van Damme S, Delrue L, Verstraete K. Well differentiated papillary mesothelioma. JBR-BTR 2007;90:541 